esterase activity in nerve fibers was detected from the axon hillock to the periphery. Beta-glucuronidase reaction was confined to nerve fibers and was negligible in ganglion cells and mantel cells.

\title{
Summary
}

Dehydrogenases participated in the citric acid cycle, isocitrate, succinate and malate dehydrogenases, were homogenously distributed in the cytoplasm or concentrated surrounding the nucleus. Glutamate dehydrogenase was also homogenously distributed in the cytoplasm of ganglion cells.

As it has been reported that sensory nerves are cholinergic in function, a varying degrees of acetylcholinesterase activity were observed in the trigeminal ganglions in the present experiment.

\section{Histochemische und radioautographische Untersuchungen von Zink im Zentralnervensystem}

\author{
Nagayasu Otsuka, Takashi Asako und Yasuhiko IBata \\ Anatomisches Institut der Medizinischen Akademie Kyoto, Kyoto
}

Mit hilfe von Dithizon konnte MASKE (1955) die Ammonsformation bei verschiedenen Säugetieren intensiv rot anfärben. Er sicherte die Spezifität für das Zink durch spektrophotometrische und emissionsspektrophotometrische Untersuchungen.

In der vorliegenden Studie soll über die Lokalisation von Zink im Zentralnervensystem mit Hilfe der Dithizoninjektion und Autoradiographie von $\mathrm{Zn}^{65}$ berichtet werden.

\section{Material und Methode}

Histochemische Untersuchung :

Es würden die Gehirne folgender Tiere untersucht: 5 Mäuse, 5 Ratten, 5 Meerschweinchen, 4 Kaninchen, 5 Katzen, 5 Hunde.

Die Tiere erhielten $100 \mathrm{mg}$ Dithizon/kg Körpergewicht in einer nach OKAMOTO wie folgt hergestellten Lösung : $100 \mathrm{mg}$ Dithizon werden mit $10 \mathrm{ml}$ 0.2\%ige Ammoniaklösung vermischt. Die dem Gewicht der Tiere entsprechend hergestelle Lösung wird sehr langsam durch eine Vene oder in die Peritonealhöhle injiziert. Etwa 15-30 min nach Beendigung der Injektion werden die Tiere durch Dekapitation getötet und die Gehirne sofort herausgenommen. Autoradiographische Untersuchung :

Zur Untersuchung gelangten die Gehirne von 20 Mäusen beiderlei Gesch- 
lechts. Das radioaktive Zink wurde in Form $\mathrm{Zn}^{65}-\mathrm{Gluconat}(50 \mu \mathrm{c})$ intraperitoneal injiziert. Die Tiere wurden in einer Spanne von 15Min., 30Min., 1Std., 6Std., 12Std., 24Std., 2Tage, 5Tage und 10Tage nach der einmaligen Injektion durch Dekapitation getötet. Die Gehirne würden sofort in abs. Alkohol fixiert, und es würden Paraffinschnitte angefertigt. Die Autoradiographie wurde mit der Stripping-Film-Methode ausgeführt.

\section{Befunde}

Die Gehirne aller untersuchten Tiere zeigen nach Dithizoninjektion eine so gleichartige Reaktion, daß wir sie gemeinsam besprechen wollen.

In der Gehirnen dieser Tiere reagieren stets die gleichen homologen cytoarchtektonischen Rindenfelder, d.h. Feld $h_{3}, h_{4}, h_{5}$ und Fascia detata der Ammonsformation nach der Dithizoninjektion stark positiv. Außer den gefärbten Abschnitten der Hippocampusformation weisen die basale Rinde und ein Teil der subcorticalen Kerne Rotfärbung auf. Bei den angefärbten Gebieten handelt es sich um den Nucl. amygdalae und Teile des Striatum. Die dorsale Hemisphärenrinde und das Hypothalamusgebit werden schwach rot gefärbt. Dagegen bleiben das Klein-, Mittel-und Rautenhirn vollkommen ungefärbt. 1 Stunde nach der Injektion von Radiozink $\mathrm{Zn}^{65}$ wird das Zink allmählich in den Nucl. amygdalae, Teile des Striatum, Hemisphärenrinde des Großhirns und Hypothalamusgebieten aufgenommen. Zwischen 12-24 Studen nach der Radioisotopgabe wird das $\mathrm{Zn}^{65}$ in den oben beschriebenen Gebieten hochkonzentriert. Jedoch in diesen Gebieten man 5-10 Tage der Gabe nur noch sehr wenig $\mathrm{Zn}^{65}$.

\section{Diskussion}

Mit Hilfe von Dithizon kann man Schwermetallsalze, unter bestimmten Bedingungen vorzugsweise Zink, nachweisen. Nach der Injektion einer Dithizonlösung färben sich bei Säugetieren bestimmte Anteile des Ammonshornes intensiv rot (MASKE 1955, FLEISCHHAUER et al. 1957, OTSUKA et al. 1962 und ORTMANN 1964).

Ferner ist es unzweifelhafte Tatsahe, daß diese bestimmten Anteile der Ammonsformation durch spektrophotometrische, emissionsspektrophotometrische (MASKE 1955) und autoradiographische (Von EULER 1962, OTSUKA et al. 1965) Untersuchungen Zink reichlich enthalten. Man bemerkt außer der Färbung im Bereich der Ammonsformation mit Dithizon eine dunkel Tönung der Großhirnrinde, des Nucleus amygdalae, des Striatum und des Hypothalamus. Auch bei autoradiographischer Untersuchung wurde das $\mathrm{Zn}^{65}$ in diesen Gebieten angereichert. Die Anhäufung von $\mathrm{Zn}^{65}$ ist nicht bleibend, sondern verschwindet nach 5-10 Tagen Speicherung wieder aus den obigen Bereichen. Jedoch sind chemische Bindung und physiologische Bedeutung des Zinks heute noch völlig unklar.

\section{Zusammenfassung}

Mit Hilfe der Dithizon-Methode und dem autoradiographischen Nachweis 
von $\mathrm{Zn}^{65}$ wurde die Lokalisation von Zink im Zentralnervensystem bei Maus, Ratte, Meerschweinchen, Kaninchen, Katze und Hund untersucht.

1) In den Gehirnen aller untersuchten Tiere färben sich die bestimmten Feldern der Ammonsformation, die Großhirnrinde, der Nucleus amygdalae, das Striatum und der Hypothalamus nach Dithizoninjektion rötlich.

2) Organisches radioaktives $\mathrm{Zn}^{65}$ wurde innerhalb von 12-24 Stunden nach der Injektion in den obigen Gebieten des Gehirns der Maus hochkonzentriert.

\title{
Literatur
}

1) Fleischhauer, K.u.E. Horstmann : Z. Zellforsch. 46, 598, 1957. 2) Maske, H.: Naturwiss. 42, 424, 1955. 3) Ortmann, N. : Progress in Brain Research Vol. 6, 4, 1964. 4) Otsuka, N. \& M. Umetani : Proc. Jap. Histochem. Ass. 3, 231, $1962 . \quad$ 5) Otsuka, N.u.M. Kawamoto: Histochmie. 6, 267, 1966.6 6) Von Euler, C. : Zit. nach Ortmann 1964.

\section{Electronmicroscopic Demonstration of Alcohol Dehydrogenase Activity in the Frog Retina}

\author{
Shozo Nishida and Katsuyoshi Mizuno \\ Department of Ophthalmology, Nagoya City University Medical School, Nagoya
}

Studies on the synthesis and breakdown of rhodopsin has been elucidated by Wald, Morton and others during a last decade. It has been confirmed that retinene reductase is identical with alcohol dehydrogenase (ADH). Though $\mathrm{ADH}$ activity in the retina has been studied biochemically and histochemically, a localization of this enzyme is not certain and the ultramicroscopic localization is essential problem. In the present paper the localization of $\mathrm{ADH}$ activity in the Bull frog retina is presented.

\section{Method}

1) After dark adaptation, Bull frog eye was enucleated and prefixed with 4\% glutaraldehyde buffered with phosphate buffer ( $\mathrm{pH} 7.4)$ in ice-box. Then the retina was incubated in the reaction media which was consisted of $0.25 \%$ nitro blue-tetrazolium chloride solution: $3.0 \mathrm{ml}, 1 \%$ DPN solution : $0.7 \mathrm{ml}, 0.2 \mathrm{M}$ ethyl alcohol : $0.3 \mathrm{ml}$, for $30 \mathrm{~min}$. at $37^{\circ} \mathrm{C}$. These treatments were conducted under dim light. After postfixation with $1 \%$ osmium tetroxide solution buffered with phosphate buffer, the retina was dehydrated by ethanol and propylene oxide and embedded in Epon 812.

In control reaction medium, ethyl alcohol was substituted with distilled water. 